\title{
Development of In-Pipe Mobile Robot Using Pneumatic Soft-Actuator
}

\author{
Mitsuhiko KUBOTA* and Toshiro NORITSUGU* \\ ${ }^{*}$ Department of Systems Engineering, Faculty of Engineering \\ Okayama University \\ 3-1-1 Tsushimanaka, Okayama, 700-8530 Japan \\ E-mail: kubota@mcrlab.sys.okayama-u.ac.jp toshiro@sys.okayama-u.ac.jp
}

\begin{abstract}
In this paper, soft-actuators are developed, which are made of a silicon rubber and are driven with a pneumatic power. The silicon rubber has a flexibility, and the pneumatic power has a compressibility. Using these characteristics, soft-actuators can be realized. A rubber can be molded in some shapes and designs, and rubber actuators can move in extending and bending.

An in-pipe mobile robot is composed of two holding actuators and a traveling actuator. The robot is fixed by hoiding actuators on both sides, a traveling actuator is equipped between holding actuators. The influence of the tube length(from servo valve to robot) is compensated by an improved control system. The compensator comprises of expresses the transfer function which is identified characteristics of the robot. Owing to the softness of the actuator and the pneumatic compliance, the robot can travel in an arbitrary shape pipe, such as a circle or a non-circle. The validity of this robot is confirmed through some experiments.
\end{abstract}

\section{KEY WORDS}

In-pipe Mobile Robot, Pneumatic Soft-actuator, Silicon Rubber, Pressure Control

\section{INTRODUCTION}

In this paper, soft-actuators are developed, which are made of a silicon rubber and are driven with a pneumatic power. The silicon rubber has a flexibility, and the pneumatic power has a compressibility. A soft-actuator can be developed by using these characteristics.

Various actuators coripposed of the rubber, such as FMA[1], HRA[2], have been developed and effectively used. The rubber can be molded in some shapes and designs, and rubber actuators can operate in extending and bending. In this paper, two types of actuators are made of the silicon rubber. The development and the control of an in-pipe mobile robot are discussed.
An in-pipe mobile robot has been expected, for example, as a mending robot, to check and operate in a pipe in the factory. There are various types of robots. Some in-pipe mobile robots are driven with the fluid power[3][4][5]. Some robots are composed of bellows actuators which are used as a holding mechanism and a driving mechanism. The fluid actuator has a high power/weight ratio and is suitable for a miniaturization. It has been reported to travel in a pipe of diameter $4[\mathrm{~mm}] \sim 5[\mathrm{~mm}][3]$. But, there is few research about a small size in-pipe mobile robot which can travel in a pipe with an arbitrary and not constant sectional diameter.

A pipe has not necessarily constant shape or size in general. There are various types of pipes, such as 


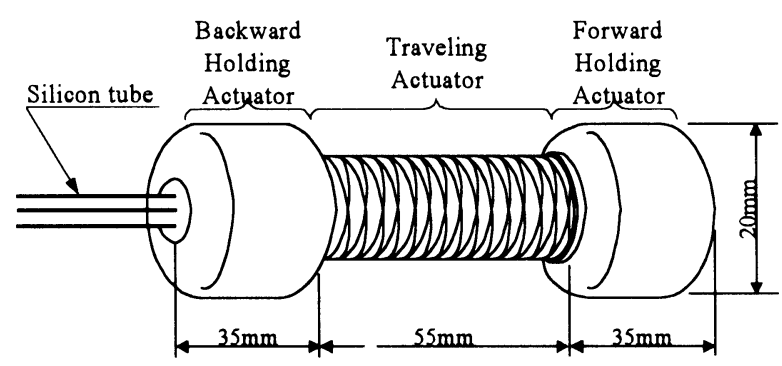

Figure 1: Structure of robot

changing diameter at a joint, wiring in a pipe, and an arbitrary shape pipe. Therefore, it is necessary to develop the robot which can travel in them. In this paper, the in-Pipe mobile robot is composed of the silicon rubber which has a high elastic characteristic, and the air pressure is used for the drive.

\section{STRUCTURE OF ROBOT}

Figure 1 shows the structure of robot. The in-pipe mobile robot is composed of two holding actuators and a traveling actuator. The robot is fixed by holding actuators on both wall sides, the traveling actuator is equipped between holding actuators. Inner pressure of each actuator is supplied through a silicon tube(outer diameter $3[\mathrm{~mm}]$, inner diameter $2[\mathrm{~mm}])$. A mass of the robot is $12[\mathrm{~g}]$. Because the robot doesn't have an electric device, it is not affected by electric and magnet noise.

\section{PNEUMATIC SOFT-ACTUATOR}

\section{OUTLINE OF ACTUATOR}

Soft actuators are used as parts of the robot. Two types of actuators are developed. One is a holding actuator that keeps the robot on a wall of the pipe, and the other is a traveling actuator that expands and contracts. These actuators are formed by the silicon RTV rubber. The silicon rubber is hardened by mixing a coagulating agent. The characteristic of silicon rubber, such as elastic modulus, elongation, and tensile strength, are changed by mixing the RTV thinner(diluent).

\section{STRUCTURE OF HOLDING ACTUATOR}

The holding actuator is expanded by supplying air pressure, and generates the force for a wall of the pipe. Therefore the robot can keep a position in the pipe.

Figure 2 shows the structure of the holding actuator. The holding actuator is composed of two hemispherical silicon rubbers, and is connected with the silicon tube to supply the pressure. The body of the actuator is expanded by the air pressure. Figure 3 shows the characteristic of the holding actuator. It is

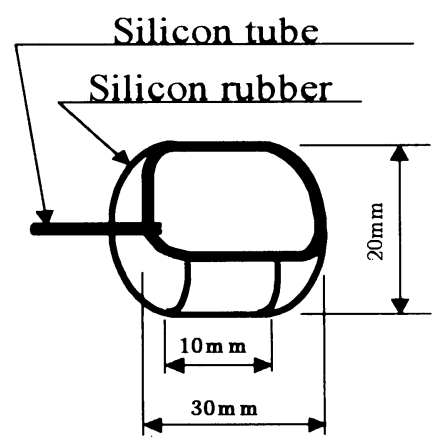

Figure 2: Structure of holding actuator

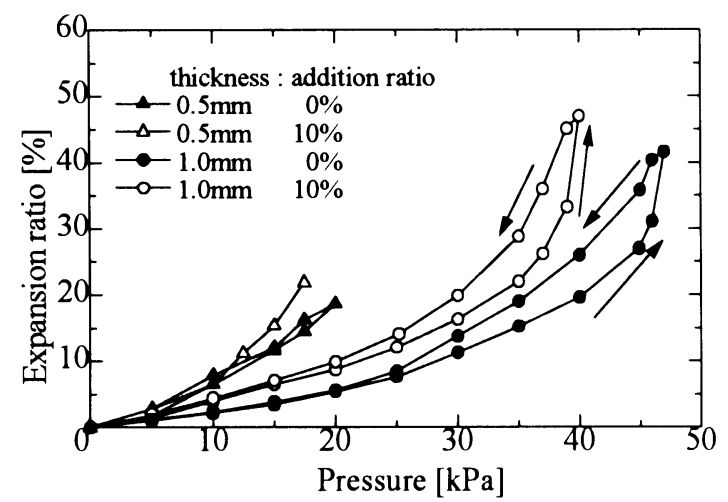

Figure 3: Static characteristic of holding actuator

a relation between the pressure and the expansion ratio(amount of diameter expansion/initial diameter). Actuators are made under $\mathbf{4}$ forming conditions, each of thickness $0.5[\mathrm{~mm}], 1[\mathrm{~mm}]$, additional ratio of RTV thinner 0[\%], 10[\%]. The actuator with the ratio of RTV thinner 10[\%] is expanded longer than one of $0[\%]$ at low pressure. The characteristic of the actuator has a hystersis and is non linear. Because of the difference of adhesion condition of the silicon rubber and so on, the holding actuator has a certain individual properties.

STRUCTURE OF TRAVELING ACTUATOR The Robot can be traveled by an elongating motion of the traveling actuator. The actuator is expanded by supplying an air pressure, and is contracted by the elastic force of silicon rubber.

Figure 4 shows the structure of the traveling actuator. A silicon tube is closely wrapped with a fiber code. Both sides of the silicon tube are closed by silicon rubbers(thickness $4[\mathrm{~mm}]$ ). The silicon rubber of the closed part isn't expanded even when applying pressure which is enough thicker than the silicon tube. The silicon tube is restricted to expanded to the radial, and is elongated only to the axial direc- 


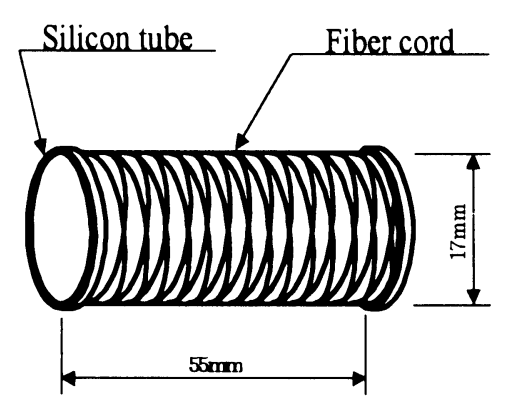

Figure 4: Structure of traveling actuator

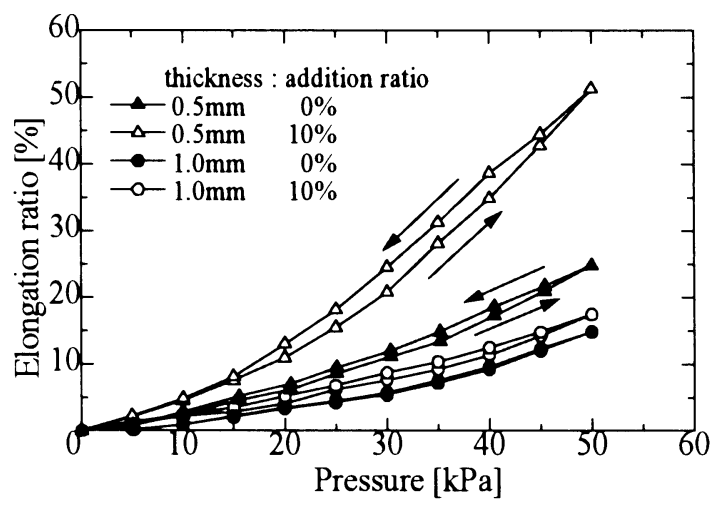

Figure 5: Static characteristic of traveling actuator

tion. Figure 5 shows the characteristic of the traveling actuator. It is a relation between the pressure and the elongation ratio(amount of elongation/initial length). As the same of the holding actuator, the actuators are made under 1 forming conditions, each of thickness $0.5[\mathrm{~mm}], 1[\mathrm{~mm}]$, additional ratio of the RTV thinner 0[\%], 10[\%]. The actuator with the thickness of $0.5[\mathrm{~mm}]$ is elongated longer than one of $1[\mathrm{~mm}]$. And the higher ratio of the RTV thinner, the longer the actuator is elongated. The characteristic of the travailing actuator has a hystersis more or less, and is a comparatively linear.

\section{TRAVELING PRINCIPLE}

Figure 6 shows a traveling principle of the robot. Figure 6(a) shows a motion of the robot. The robot can move to the right by repeating (1) (6). (1) (6) motions are as followed.

(1) The backward holding actuator is expanded, the forward holding actuator is contracted.

(2) The backward holding actuator is expanded, the traveling actuator is elongated.

(3) The forward holding actuator is expanded.

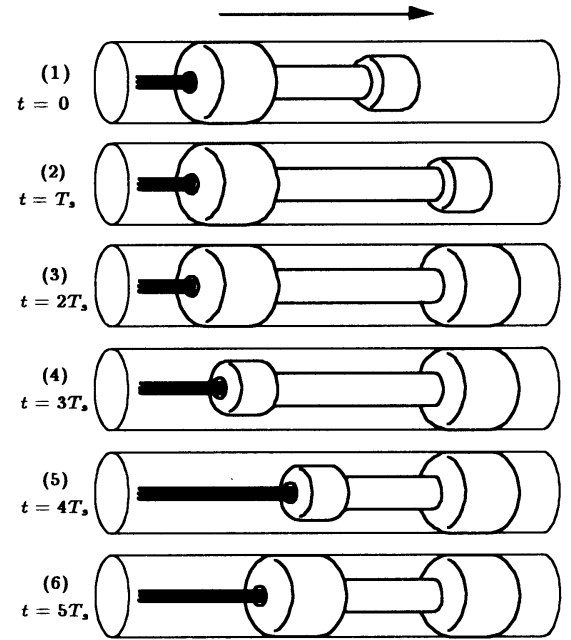

(a) Action of robot

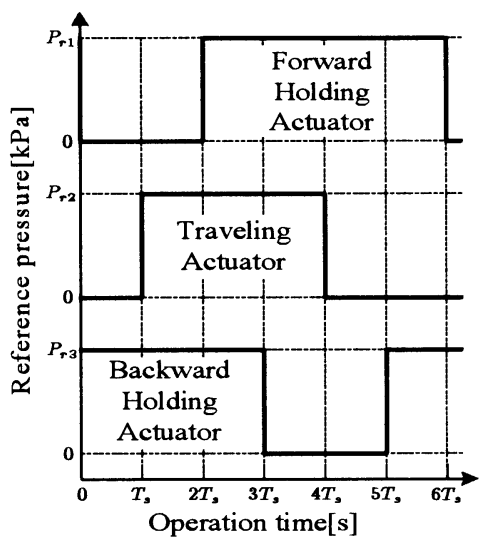

(b) Traveling method

Figure 6: Traveling method

(4) The forward holding actuator is expanded, the backward holding actuator is contracted.

(5) The traveling actuator is shortened.

(6) The backward holding actuator is expanded.

In case of traveling to the left, The motion is reversed as (6) (1). Figure 6(b) shows the reference pressure of each step. $P_{r 1 \sim 3}$ are reference pressure of each actuator which aren't necessary to be so precisely controlled. $T_{s}$ is the unit time to change the motion of robot. A traveling speed of robot can be changed by $T_{s}$. The pressure of each actuator is controlled by a PI controller. Considering the bonding strength on the silicon tube, the maximum reference pressure of each actuator is set lower than $40[\mathrm{kPa}]$. 


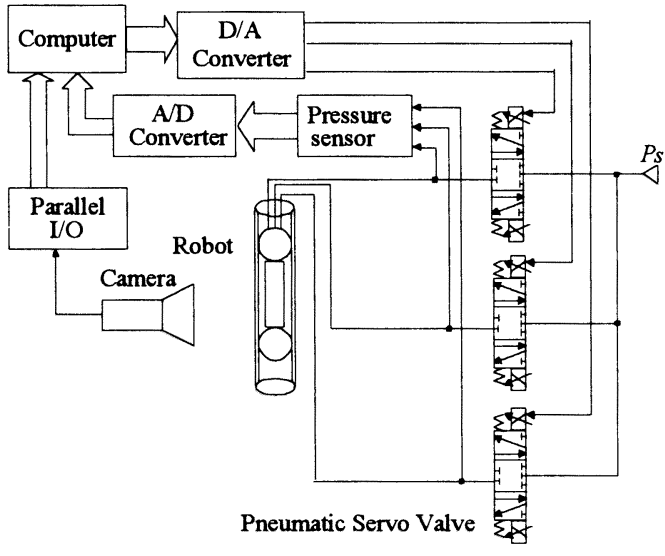

Figure 7: Experinent apparatus

\section{EXPERIMENT APPARATUS}

Figure 7 shows an experiment apparatus. Each of the actuator and the pneumatic servo valve are connected by a silicon tube. The inner pressure of actuator is measured with pressure sensors near the output port of the servo valve. The supply pressure is $P_{s}=100[\mathrm{kPa}]$, the air is exhausted from the actuator to the atmosphere. The position of the robot in a transparent pipe is measured with a camera.

\section{CONTROL SYSTEM}

In case of traveling and working in the pipe, it is desirable that the robot can travel a long distance. The output pressure from the servo valve is transferred to the robot through the silicon tube. When the length of pressure supply tube becomes long, the pressure response of the actuator delays. This section describes a compensation method for a delay of the pressure response due to the length of pressure supply tube.

Figure 8 shows an experiment apparatus. Pressure sensors are set up near the output port of the servo valve and near the robot. $P_{1}$ is a pressure value from the sensor 1. $P_{2}$ is a pressure value from the sensor 2. If it is possible to measure $P_{2}$, pressure control system shown in Figure 9 can be constructed. Where, $P_{r}$ is a reference pressure. $C$ is a controller. $u$ is a driving value of the servo valve. $G$ is a transfer function that means the pressure response of the actuator with the pressure supply tube. However, when the robot travels in pipe, the body of the robot becomes larger to set up a normal size sensor. Other case, when attaching a small sensor to the actuator, an elasticitically property of the robot is lost. Therefore, each actuator is controlled based on the sensor 1 which is set near the output of the servo valve.
Table1: Time constant $T(s)$

\begin{tabular}{|c|c|c|c|}
\hline & $\begin{array}{c}\text { Forward } \\
\text { holding } \\
\text { actuator }\end{array}$ & $\begin{array}{c}\text { Traveling } \\
\text { actuator }\end{array}$ & $\begin{array}{c}\text { Forward } \\
\text { holding } \\
\text { actuator }\end{array}$ \\
\hline 3m & 0.06 & 0.04 & 0.045 \\
\hline $5 \mathrm{~m}$ & $\mathbf{0 . 0 9}$ & $\mathbf{0 . 0 8 2}$ & $\mathbf{0 . 0 8 2}$ \\
\hline $7 \mathrm{~m}$ & $\mathbf{0 . 1 7}$ & $\mathbf{0 . 1 5}$ & $\mathbf{0 . 1 5}$ \\
\hline
\end{tabular}

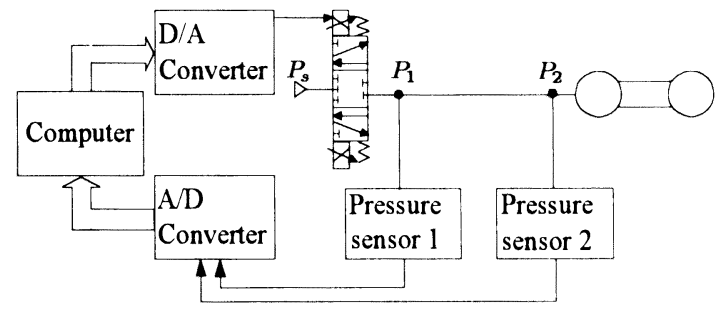

Figure 8: Experiment apparatus

Firstly, a gain of the controller $C$ is determined by using the sensor 2 in the control system Figure shown 9. Secondly, the transfer function $G_{m}$ is identified by means of comparing the pressure response from the sensor 1 with one from the sensor 2 . The transfer function is obtained from the result of experiments as follows:

$$
G_{m}=\frac{e^{-s L}}{1+s T}
$$

$L$ is a dead time(length of supply tube)/(velocity of sound). Table 1 shows the time constant $T$ identified of length for the pressure supply tube $3[\mathrm{~m}], 5[\mathrm{~m}]$, and $7[\mathrm{~m}]$. By using the transfer function $G_{m}$, the pressure control system is built up by using the sensor 1 as shown in Figure 10. If $G_{m}$ is a correct, Figure 9 and Figure 10 are equivalent. The pressure control of $P_{2}$ can be carried out by a closed loop system using $P_{1}$. Although $G_{m}$ has a dead time, as this robot repeats the step of Figure 6, the pressure response shifted only dead time does not influence the robot motion.

\section{RESULTS AND DISCUSSION}

\section{TRAVELING WITH PAYLOAD}

The robot travels in the transparent pipe which is set in the vertical. Figure 11 shows an experimental result. The robot travels with a payload in the pipe of the diameter of $24[\mathrm{~mm}]$. The length of the pressure supply tube is $1[\mathrm{~m}]$. The reference pressures of the actuators are $P_{r 1}=39[\mathrm{kPa}], P_{r 2}=40[\mathrm{kPa}]$ and $P_{r 3}=39[\mathrm{kPa}]$. As $T_{s}$ is set to $100[\mathrm{~ms}]$, the motion cycle $(1) \sim(6)$ in Figure 6 is repeated every $600[\mathrm{~ms}]$. 


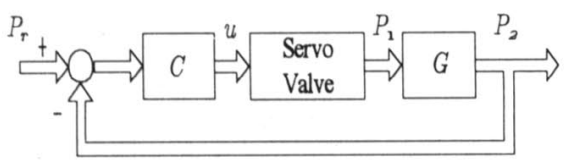

Figure 9: Bloack diagram of pressure control 1

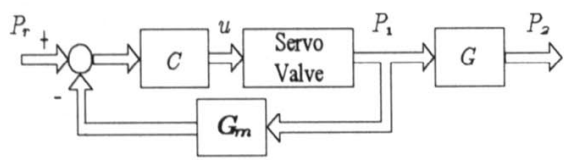

Figure 10: Bloack diagram of pressure control 2

When the length of supplying tube is $1[\mathrm{~m}]$, the delay of pressure is so small that $G_{m}=1$ in Figure 10 .

The average traveling speed is $13[\mathrm{~mm} / \mathrm{s}]$ without the payload, and is $44[\mathrm{~mm} / \mathrm{s}]$ with the payload of $250[\mathrm{~g}]$. As the traveling actuator is elongated to downward by the payload at the step (5) in Figure 6, the traveling speed changes with the payload. As the traveling actuator is elongated longer than its stroke by the payload over $290[\mathrm{~g}]$, the robot cannot move.

\section{CHANGE OF PIPE SECTIONAL SHAPE}

Figure 12 shows a photograph of traveling in a triangle pipe. It is a regular triangle and a side of triangle is $44[\mathrm{~mm}]$. Figure 13 shows a photograph of traveling in the pipe of which diameter changes from $24[\mathrm{~mm}]$ to $28[\mathrm{~mm}]$. Conversely, the robot can travel when the diameter of pipe changes from $28[\mathrm{~mm}]$ to $24[\mathrm{~mm}]$.

As the holding actuator can be adaptively changed its shape owing to the flexibility of silicon rubber and the compressibility of pneumatic pressure, the robot can travel in these pipes.

\section{CHANGE OF SUPPLY TUBE LENGHT}

The length of supply tube from valve to the robot changes to $3[\mathrm{~m}], 5[\mathrm{~m}]$ and $7[\mathrm{~m}]$. The robot is moved in the pipe with the diameter of $24[\mathrm{~mm}]$ without payload. Figure 14 shows experimental results. The reference pressures of actuators are $P_{r 1}=36[\mathrm{kPa}]$, $P_{r 2}=40[\mathrm{kPa}]$ and $P_{r 3}=35[\mathrm{kPa}]$. The reference pressures $P_{r 1}$ and $P_{r 3}$ of the holding actuators are the lowest pressure to travel in the pipe with the diameter of $24[\mathrm{~mm}]$ without a payload.

When the length of pressure supply tube is $3[\mathrm{~m}]$, $T_{s}$ is set to $100[\mathrm{~ms}]$. As same as the result of a length of $1[\mathrm{~m}]$, The robot can travel at the speed of $13[\mathrm{~m} / \mathrm{s}]$.

When the length of the supply tube is $5[\mathrm{~m}]$, the traveling speed is measured in two case where the pressure $P_{1}$ is compensated by $G_{m}$ as shown Figure 10 and not compensated. When the control system is compensated by $G_{m}$, the robot can travel for

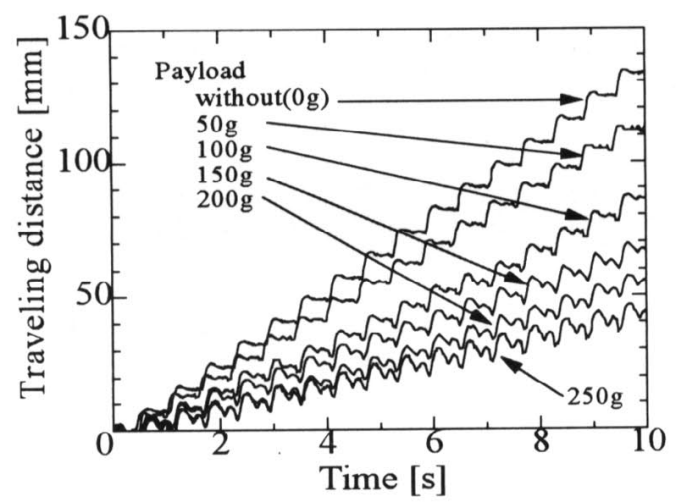

Figure 11: Traveling distance of robot

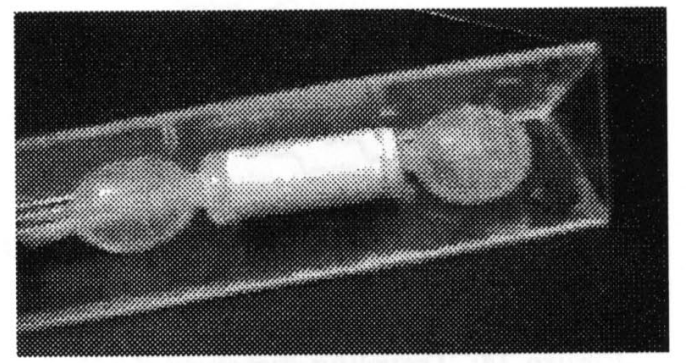

Figure 12: Photo of traveling in triangle pipe

$T_{s}=100[\mathrm{~ms}]$. When it is not compensated, $G_{m}=1$, the response of the actuator largely delays. Therefore, $T_{s}$ is necessary to be made larger to travel without the compensation, but the traveling speed becomes small.

When the length of supply tube is $7[\mathrm{~m}], T_{s}$ is set to $125[\mathrm{~ms}]$. Figure 15 shows the pressure response of each actuator. $P_{1}$ is compensated by $G_{m}$. The pressure response of $P_{1}$ and $P_{2}$ are measured by the pressure sensors. The pressure response of $P_{2}$ has a dead time when the reference pressure changes. The traveling speed is becomes small by setting $T_{s}=125[\mathrm{~ms}]$. When the reference pressure of the traveling actuator changes from the reference pressure to $0[\mathrm{kPa}]$, the reference pressure changes before the pressure in actuator perfectly reaches $0[\mathrm{kPa}]$. Also this response delay makes the speed smaller due to the reduction of the traveling stroke. When $T_{s}$ is set to a small value, the motion period becomes short, but the stroke of the robot is reduced by the pressure response delay of the traveling actuator. When $T_{s}$ is set to a large value, the motion period becomes large, but the robot can move with a longer stroke.

\section{CONCLUSION}

In this paper, a pneumatic soft-actuators are devel- 


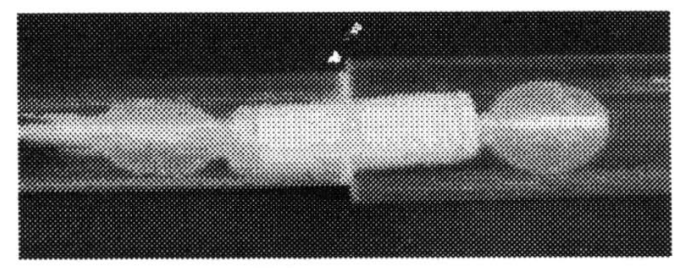

Figure 13: Photo of traveling in changing diameter of pipe

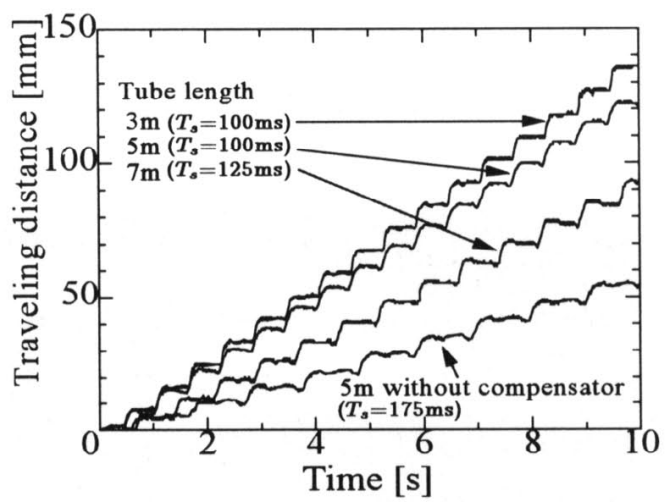

Figure 14: Traveling distance of robot

oped by using the silicon rubber, and an in-pipe mobile robot is composed of them. Results are followed.

(1) An in-pipe mobile robot is developed, which are made of the silicon rubber and is driven with the pneumatic power. The holding actuator can adaptively change its shape owing to the flexibility of the silicon rubber and the air pressure. Therefore, the robot can travel in the pipe with an arbitrary sectional shape.

(2) When the length of pressure supply tube from an air servo valve to the robot is long, the pressure response of each actuator is delay. The pressure response of the actuator with supply tube is identified as a transfer function, and the control system is compensated by the transfer function. As a result, the motion performance of robot can be improved, also the reductionof the traveling speed of robot can be avoided.

\section{References}

[1] K.Suzumori, Flexible Microactuator, Journal of The Japan Society of Mechanical Engineers, 56527, pp.1887-1892 1990.

[2] S.Kawamura et.al., Development of a Hexahedron Rubber Actuator(HRA), Journal of

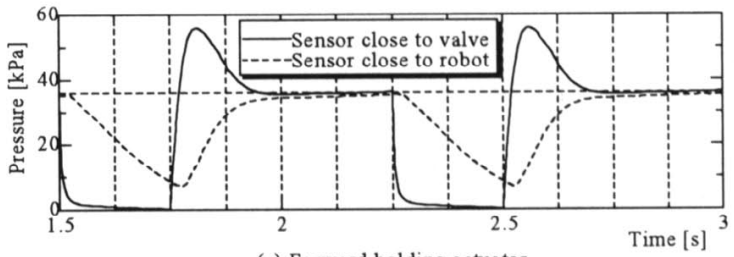

(a) Forward holding actuator

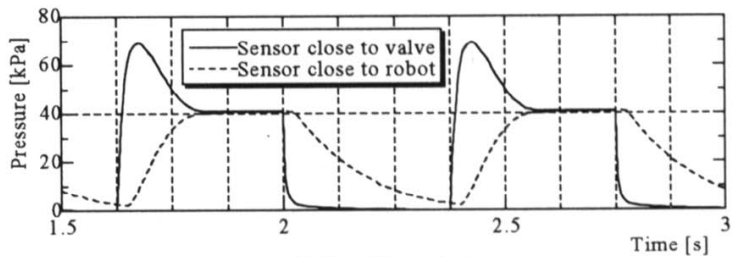

(b) Traveling actuator

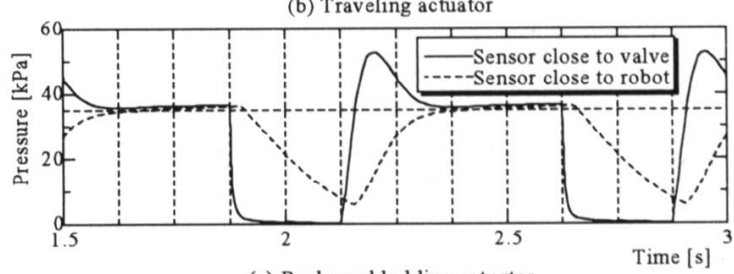

(c) Backward holding actuator

Figure 15: Response of pressure(Tube length $7[\mathrm{~m}]$ $\left.T_{a}=125[\mathrm{~ms}]\right)$

Robotics Society of Jpan, vol.16, No.3, pp.369375, 1998.

[3] K.Yoshida S.Yokota. An In-Pipe Mobile Micromachine Using Fluid Power, Journal of The Japan Hydraulics and Pneumatics Society, 292, pp.179-184, 1997.

[4] S.Kato T.Hirayama, Fabrication of Artificial Earthworms Driven By Pneumatic Pressure, Proc. Mechanical Engineers, pp.6616741997.

[5] K.Suzumori S.Sekiguchi, Pneumatic Rubber Actuator Driven by Surface Traveling Waves, Proc. Mechanical Engineers, pp.6758761997. 